doi) https://doi.org/10.31977/grirfi.v21i3.2393

Recebido: 24/05/2021 | Aprovado: 15/09/2021

Received: 05/24/2021 | Approved: 09/15/2021

\title{
EL PROBLEMA DEL LENGUAJE EN HEGEL: TRES LECTURAS DE LA ENCICLOPEDIA DE LAS CIENCIAS FILOSÓFICAS (1817)
}

\author{
Federico Gabriel Cortés ${ }^{1}$ \\ Universidad Nacional de La Plata (UNLP - CONICET) \\ https://orcid.org/0000-0003-1678-1262 \\ E-mail: fede.gcortes@gmail.com
}

\section{RESUMEN:}

En este trabajo retomamos una serie de lecturas sobre la Enciclopedia de las ciencias filosóficas (1817) -texto tardío de la obra de Hegel, en el que presenta su sistema filosófico de manera completa y acabada-: La respiración del ser de Germán Prósperi, "Hegel's Linguistic Thought in the Philosophy of Subjective Spirit. Between Kant and the Metacritics" de J. Surber y "Sign and Symbol in Hegel's Aesthetics" de Paul de Man. Proponemos hacer hincapié en tres instancias que Hegel analiza en el apartado sobre "El espíritu subjetivo": el recuerdo, la imaginación y la memoria. También haremos referencia a Ciencia de la lógica (1812) porque allí Hegel desarrolla la especial relevancia de la dialéctica para el pensamiento filosófico. Nuestro objetivo es vincular el tratamiento hegeliano de las tres instancias mencionadas con sus teorizaciones en torno al problema de la nominación y el lenguaje, buscando indagar en las tensiones que estas conceptualizaciones suponen al interior de su sistema filosófico.

PAlABRAS CLAVE: Lenguaje; Hegel; Resistencia; Dialéctica.

\section{THE PROBLEM OF LANGUAGE IN HEGEL: THREE READINGS FROM THE ENCYCLOPEDIA OF PHILOSOPHICAL SCIENCES (1817)}

\begin{abstract}
:
In this work we review a set of readings of Encyclopedia of the Philosophical Sciences (1817) -late text of Hegel's work, in which he presents his philosophical system in a completed and finished manner-: La respiración del ser from Germán Prósperi, "Hegel's Linguistic Thought in the Philosophy of Subjective Spirit. Between Kant and the Metacritics" from J. Surber y "Sign and Symbol in Hegel's Aesthetics from Paul de Man. We propose to emphasize in three aspects analyzed by Hegel in the section on the "Subjective Spirit": memory, imagination and remembrance. We will also make reference to Science of Logic (1812) in which Hegel develops the importance of dialects for philosophy. Our aim is to relate the three aspects mentioned above with Hegel's theorizations of language and nomination, in order to stress that these conceptualizations implies within his philosophical system.
\end{abstract}

KEYWORDS: Language; Hegel; Resistance; Dialectics.

\footnotetext{
${ }^{1}$ Doutorando(a) em Letras na Universidad Nacional de la Plata (UNPL - CONICET), Buenos Aires, Argentina.
}

CORTÉS, Federico Gabriel. El problema del lenguaje en Hegel: tres lecturas de la Enciclopedia de las ciencias filosóficas (1817). Griot : Revista de Filosofia, Amargosa - BA, v.21 n.3, p.258-267, outubro, 2021. 


\section{Introducción}

Antes de comenzar con el análisis de los textos, buscaremos recuperar brevemente algunas formulaciones de Hegel que son de interés para nuestro trabajo, tomando como referencia el texto Essays on Hegel's Philosophy of Subjective Spirit (2013) editado por David S. Stern. En la introducción al libro, Stern destaca que esta sección de la obra hegeliana es una de las áreas menos estudiadas y conocidas de la filosofía de Hegel: "Antes de 1970 virtualmente no existían estudios sobre la Filosofía del espíritu en inglés" (STERN, 2013, 9). Nos interesa particularmente este dato histórico porque en el texto que analizaremos de Paul de Man, previo a las fechas mencionadas por Stern pero en el ámbito de la teoría literaria y no de la filosofía, se retoman varios parágrafos de esa sección.

Hegel comienza la sección sobre la Filosofía del espíritu afirmando que "en el alma se despierta la conciencia; la conciencia se pone como razón que está inmediatamente despierta respecto al saber de sí y que mediante su actividad se libera en orden a la objetividad y a la conciencia de su concepto" (HEGEL, 2005, 440). El espíritu, al igual que el concepto, se desarrollan progresivamente hacia su meta: "hacerse y devenir para sí aquello que él es en sí" (HEGEL, 2005, 440). La dialéctica es la ley que gobierna este movimiento por medio del cual el alma (entendida como el en-sí inicial del espíritu) se convierte en conciencia (entendida como el para-sí del espíritu), quedando disponible como objeto de conocimiento. Recordemos que luego de haber publicado la Fenomenología del espíritu (1807) Hegel se propone establecer la ciencia como lógica en su texto Ciencia de la lógica de 1812. Allí establece que la dialéctica no es un apartado de la lógica sino que son equivalentes, homologables. El método, dice Hegel, "no es en nada distinto a su objeto y contenido; pues es el contenido dentro de sí mismo, la dialéctica que él en sí contiene, lo que lo mueve hacia adelante" (HEGEL, 2011, 203). De esta manera Hegel ontologiza la lógica, la convierte en ser, cuando afirma que la dialéctica no "es un hacer exterior y negativo que no pertenece a la cosa misma" (HEGEL, 2011, 204).

\section{La dialéctica y el alma}

En La respiración del ser (2018) Germán Prósperi estudia la sección de la Enciclopedia titulada "Antropología" -cuyo objeto es el "alma". Su argumentación comienza por considerar que este objeto "constituye de algún modo su núcleo gravitatorio y a la vez su mancha ciega" (PRÓSPERI, 2018, 17). Si el movimiento dialéctico de la "idea" en Hegel consiste en salir hacia la "naturaleza" y luego recobrarse en el "espíritu", para Prósperi la particularidad del objeto "alma" radica en que "no es ya el medio a la vez espiritual y natural que asegura la respiración del ser, sino el intersticio, $n i$ natural $n i$ espiritual (es decir neutro: ne... uter) que suspende el movimiento dialéctico y lo disloca por completo" (PRÓSPERI, 2018, 14). El "alma" en tanto objeto que no ha alcanzado la conciencia - objeto que todavía no es un "yo" - configura un lugar imposible, un punto límite en la medida en que es un objeto inherentemente ambiguo que, según Prósperi, señala "la imposibilidad del espíritu y de la naturaleza, el instante intangible que pareciera opacar el pensamiento eficiente de la dialéctica" (PRÓSPERI, 2018, 18).

A lo largo del libro, Prósperi analiza cuatro fenómenos específicos que aparecen desarrollados en la Enciclopedia: el niño en el vientre materno, el sonambulismo, la locura y el sueño. Prósperi parte de la hipótesis de que el alma puede pensarse como "imaginación", recurriendo por un lado a J. Reid para demostrar que la imaginación interviene en los cuatro fenómenos mencionados, y por otro lado a G. Bataille, quien en La experiencia interior (1943) 
nombró como "mancha ciega" a aquel "punto o límite que no puede ser asimilado por la lógica dialéctica" (PRÓSPERI, 2018, 35). De esta manera, su apuesta pasa por leer el espacio de la imaginación como ese punto inasimilable por el pensamiento dialéctico, en la medida en que funciona como "articulación de las grandes dualidades metafísicas: lo sensible y lo inteligible, el cuerpo y el alma, la naturaleza y el espíritu" (PRÓSPERI, 2018, 28). Entonces, si desde el punto de vista de la lógica, Hegel concibe la posibilidad de la "idea" como un devenir, como un desarrollo en el que la idea debe salir de sí, volver a la naturaleza, para luego recuperarse en el espíritu y poder alcanzar el autoconocimiento -figurable, alegóricamente, como los movimientos de inhalación y exhalación del proceso respiratorio de la "respiración de la Idea"-, Prósperi utiliza la figura de la "apnea" para describir la interrupción/suspensión de este proceso dialéctico, destacando el lugar del "alma" como su condición de (im)posibilidad:

\begin{abstract}
el proceso dialéctico [...] es la respiración de la Idea, en sus dos movimientos: expiración en la naturaleza, inspiración en el espíritu, exhalación material, inhalación material. El alma [...] es el medio que garantiza el tránsito de un movimiento a otro [...] entendida como suspensión o pausa, como hendidura neutra (ni material $n i$ espiritual) como apnea (PRÓSPERI, 2018: 46).
\end{abstract}

Ahora bien, recordemos que la organización de la sección "El espíritu subjetivo" de la Enciclopedia está dividida en tres: la "Antropología", la "Fenomenología del espíritu" y, por último, la "Psicología", dedicadas al alma, la conciencia y espíritu respectivamente. De esta manera, queremos notar que la propuesta de Germán Prósperi, consistente en relacionar el alma con la imaginación, es un recorrido que va desde la sección sobre el alma a la sección sobre el espíritu - sección fundamental para nuestro trabajo porque contiene una de las pocas argumentaciones sistemáticas de Hegel sobre el tema del lenguaje-. En el mencionado libro Essays on Hegel's Philosophy of Subjective Spirit, el texto dedicado al lenguaje se titula "Hegel's Linguistic Thought in the Philosophy of Subjective Spirit. Between Kant and the Metacritics" escrito por J. Surber. Allí se reconstruye y analiza el apartado que Hegel le dedica a la "Representación", incluido en la sección "Psicología". Este apartado es sumamente importante para nuestro trabajo porque allí la Representación se aborda siguiendo tres instancias bien delimitadas: el recuerdo, la imaginación y la memoria. Nos detendremos a continuación en el análisis de esta sección de la obra hegeliana, utilizando como referencia el mencionado texto de Surber y "Signo y símbolo en Hegel" de Paul de Man.

\title{
Lenguaje y representación
}

Tal como aparece sugerido en el título de su artículo, Surber sostiene que Hegel mantiene y completa el enfoque trascendental elaborado por Kant, confrontando con el escepticismo metacrítico de Herder y Hamann -quienes desconfiaban de la posibilidad de desarrollar una filosofía sistemática por las limitaciones propias del lenguaje para construir verdades y principios universales-. En relación con esto, la propuesta de Hegel se destaca especialmente por la instancia de la representación como punto articulador para la reflexión lógica:

\footnotetext{
Hegel considera a la significación y al lenguaje como elementos esenciales de la representación, que es el fundamento necesario para el pensamiento [...] esto constituye un distanciamiento significativo de la valoración negativa que Kant realiza del lenguaje como un asunto meramente empírico que no tiene ningún rol en la indagación trascendental" (SURBER, 2013, 188).
} 
El apartado sobre la "Representación" que, como dijimos anteriormente, se ocupa sistemáticamente del lenguaje, mediatiza entre la "Intuición" (cuyo objeto es la imagen) y el "Pensamiento" (que trata sobre el concepto). A raíz de esto, Surber establece que el abordaje dialéctico de Hegel permite dar cuenta de forma más acabada -respecto del enfoque trascendental kantiano- del rol que juegan el lenguaje y la significación en los distintos procesos de cognición.

Según Surber, esta dialéctica entre cognición y significación, que opera en la representación, es uno de los aportes más novedosos de la filosofía hegeliana. Cada facultad cognitiva tiene entonces su correlato en un producto significacional que funciona como expresión objetiva, es decir, como "contenido" de dicha facultad. A continuación nos detendremos en detallar este proceso dialéctico según aparece definido en el apartado "Representación" de la Enciclopedia de las ciencias filosóficas, teniendo en cuenta que al ser el lugar donde la imaginación se articula con el lenguaje, es posible localizar allí el punto en el que se sitúan la lectura crítica de Paul de Man.

La representación es internamente compleja en la medida en que contiene diversas facultades relacionadas entre sí. En tanto es un proceso dialéctico, puede ser descripta como el devenir de la "intuición" (es decir, del hecho de percibir por medio de los sentidos) al "concepto" (general, abstracto, que es la base del pensamiento). De esta manera, se parte de la "intuición" que produce una imagen sensible ("Bild") que no es discursiva y, por tanto, carece de la objetividad de los conceptos. La imagen sensible posee un "contenido sensible" porque es copia de la intuición sobre la que se basa, pero con la particularidad de existir en el sujeto y no como exterioridad. La siguiente instancia es la "recolección/recuerdo" ("Erinnerung") que produce una imagen física que ahora se sostiene en sí misma y no depende de la intuición, adquiriendo cierto grado de generalidad. Luego sigue la "imaginación" que se divide en tres tipos: "reproduktive Einbildungskraft", "productive Einbildungskraft o Phantasie" y "zeichenmachende Phantasie"2. La primera de ellas produce ya una representación abstracta, siendo su función la de recuperar las imágenes previas para que puedan ser expresadas. La segunda, introduce la distinción entre "signo" y "símbolo", y se destaca por combinar libremente las representaciones de la instancia anterior. El símbolo es definido como una imagen que ya no tiene un sentido inherente sino un segundo sentido producto de la unión de dos representaciones abstractas cualesquiera; es una imagen internalizada que adquiere un sentido simbólico al conectarse con otra imagen internalizada. El signo, por su parte, emerge cuando un objeto "externo" se asocia, mediante la "imaginación productiva" con una representación abstracta ya internalizada. Por último, la "imaginación creadora de signos" toma esos signos y los combina para conformar así el lenguaje como unidad mayor. Todo esto desemboca en la "memoria" (Gedachtnis) que es la facultad cognitiva que produce los "nombres". Según Hegel, éstos son el contenido determinado pero sin dependencia de la imagen como en las instancias anteriores. En el nombre, el contenido y el significado de la intuición han desaparecido, quedando el signo en sí -libre de ataduras referenciales-; de este modo, en el vacío del sentido, puede aparecer el pensamiento como verdad y objetividad, desembocando finalmente en el "concepto". Los nombres, dice Surber, "a diferencia de las palabras o los signos, son libres de imágenes y representaciones, y por lo tanto son ahistóricos, transculturales y objetivos, lo cual los convierte en el único vehículo posible para el pensamiento" (SURBER, 2013, 196).

Detengámonos aquí para medir las implicancias de este desarrollo. Si el "nombre", entendido como el signo ya despojado de la imagen e internalizado por el sujeto, funciona como

\footnotetext{
${ }_{2}^{2}$ Se pueden traducir de la siguiente manera: imaginación reproductiva, imaginación productiva e imaginación creadora de signos.
}

CORTÉS, Federico Gabriel. El problema del lenguaje en Hegel: tres lecturas de la Enciclopedia de las ciencias filosóficas (1817). Griot : Revista de Filosofia, Amargosa - BA, v.21 n.3, p.258-267, outubro, 2021. 
condición de posibilidad para la existencia del pensamiento y el "concepto", esto significa que en última instancia habría una relación de identidad entre los nombres y los conceptos. Según Surber, esto implicaría que todas las determinaciones lógicas serían nombres, lo cual entra en contradicción con la propia afirmación hegeliana de que las determinaciones del pensamiento son los "conceptos".

Es llamativo el modo en el que Surber se excusa y resiste a las conclusiones a las que él mismo llega, reduciendo la teoría de hegeliana de los nombres a una respuesta histórica. Dice Surber acerca de la contradicción que introduce esta identidad entre "nombre" y "concepto" según se deduce en el apartado "Representación":

Hegel no se refiere a este tema en la Filosofía del espíritu subjetivo ni en ningún otro escrito que yo conozca [...] En este punto sólo puedo concluir que, si la teoría de los nombres de Hegel fue de hecho una respuesta a los metacríticos (como he sugerido), genera potencialmente entonces problemas más difíciles para otras partes de su enfoque filosófico (SURBER, 2013, 196).

Destacamos esto porque consideramos que son estos "problemas" que hace emerger la consideración del lenguaje y de los nombres en Hegel, al interior de su sistema filosófico, los que son retomados por Paul de Man en el texto que analizaremos a continuación.

\section{La resistencia como tensión entre experiencia y lenguaje: Gedachtnis y Erinnerung}

El texto de De Man se publica por primera vez en el libro Aesthetic Ideology (1996), que reúne las últimas clases dictadas entre los años 1977 y 1983. Dentro del conjunto de la obra de De Man, este libro se destaca porque su corpus de análisis se compone exclusivamente de textos filosóficos. Recordemos que De Man, junto con J. Derrida, es uno de los autores más fuertemente vinculados con la "deconstrucción" en el ámbito universitario estadounidense.

El modo de lectura de De Man se caracteriza por hacer hincapié en la dimensión retórica del lenguaje como aquello que resiste a la lógica de la gramaticalización. Otro de sus rasgos más importantes, que se pone de manifiesto en toda su obra pero que se destaca especialmente en Aesthetic Ideology, es que propone un modo de lectura que metodológicamente no difiere en el abordaje de textos literarios y textos filosóficos -así, por ejemplo, en varios de sus textos lee de manera conjunta a Nietzsche con Baudelaire-. El intento demaniano por articular una reflexión conjunta del discurso literario y el filosófico alcanza entonces su culminación en Aesthetic Ideology; allí el gesto novedoso de De Man consiste en deconstruir los sistemas filosóficos de Hegel y Kant a partir de una consideración específica del lugar que en ellos ocupa la teorización sobre la "experiencia estética". A continuación, analizaremos este modo de lectura tal como se desarrolla en el texto "Signo y símbolo en Hegel", teniendo en cuenta que retoma las zonas de la obra hegeliana que estamos abordando en este trabajo.

De Man parte de la tensión entre la experiencia (literaria, estética, de lectura) y la teoría, como modo de problematizar la pretensión del método (por caso, de los estudios literarios) de aspirar a rigor teórico y generalidad pero manteniendo intacto, o incluso pretendiendo facilitar, la apreciación estética o el potencial para el análisis histórico de la obra. Su tesis es que la estética hegeliana es la afirmación más sistemática de la estructura, la historia y la valoración del arte, en la medida en que la posibilidad de la "síntesis" descansa en el poder reunificador de la categoría de la "estética": "El poder de síntesis filosófica radica en la posibilidad de la obra de reunir, bajo la protección de la estética, una causalidad histórica con una estructura lingüística, un evento 
experimental y empírico en el tiempo con un hecho, indeterminado y no fenomenal, del lenguaje" (DE MAN, 1996, 93). Esto queda plasmado en la organización de las tres fases de la historia del arte que propone Hegel: las dos primeras están signadas por términos históricos (artes "Clásico" y "Romántico"), mientras que la tercera se nombra a partir de un término lingüístico (arte "Simbólico"). ${ }^{3}$

La cuestión del símbolo es sumamente importante en la estética hegeliana, al punto tal que "la teoría de la estética, como noción histórica y filosófica, está predicada en una teoría del arte como símbolo" (DE MAN, 1996, 93). La belleza, el goce estético, es entendida según este régimen simbólico que mediatiza entre la mente y el mundo físico. Para enmarcar el problema que esto supone, De Man pone en juego dos conocidas caracterizaciones de Hegel sobre el arte: el arte es a la vez manifestación sensible de la Idea (en tanto participa de lo bello) y "Para nosotros, cosa del pasado".

De Man lee en serie estas dos formulaciones: si el arte es manifestación sensible de la Idea, y si para nosotros el arte es cosa del pasado, ¿esto significa que ya no podemos producir formas simbólicas, que la manifestación sensible de la Idea ya no es accesible para nosotros como forma? "Es una ironía que Hegel declare muerto al arte cuando el siglo XIX renueva sus formas; Hegel no puede leer a sus contemporáneos" (94). De Man propone que Hegel no puede dar cuenta del carácter metafórico de las poéticas que le son contemporáneas a causa de su inadecuada concepción del lenguaje. A partir de esto formula una pregunta retórica deconstructiva: ¿puede ser que no podamos leer lo que dice Hegel sobre el símbolo y el lenguaje porque esto desestabilizaría "el valor de la estética" (DE MAN, 1996, 95)?

De Man rastrea la distinción entre "signo" y "símbolo" planteada por Hegel en La enciclopedia (1817) y La estética (1830), para proponer que el problema radica justamente en que esta distinción no se sostendría en el arte porque excluiría todas las formas de expresión norepresentacionales. Retomando lo que vimos anteriormente, por un lado el signo "estresa la arbitrariedad de la relación entre el componente sensorial necesariamente involucrado en cualquier signo y el significado intencionado [intended meaning]" (DE MAN, 1996, 96), de manera que no hay continuidad entre el contenido de la percepción y el contenido de lo significado. Por otro lado, el símbolo es definido como "una percepción cuya propia determinación [o significado] más o menos corresponde, esencial o conceptualmente, con el contenido que expresa en tanto símbolo" (DE MAN, 1996, 96). De Man valora el signo por sobre el símbolo, en tanto el primero no depende de las propiedades referenciales a las que apunta para poder significar.

En este punto, queremos recordar que la distinción entre signo y símbolo aparece en el desarrollo dialéctico analizado anteriormente, el progreso de la intuición al pensamiento y al concepto. De Man, en un gesto funcional a la argumentación de su apuesta teórica, realiza una apropiación retórica de Hegel al decir que su filosofía consiste en una dialéctica de la internalización: "La metáfora que organiza el sistema entero es la de la interiorización" (DE MAN, 1996, 100). El arte y la literatura, entendidos como manifestación sensorial de un contenido interno- una entidad que ha sido internalizada por medio del proceso dialéctico que involucra los distintos tipos de "imaginación" analizados ${ }^{4}-$, es en sí misma también un contenido externo. De

\footnotetext{
${ }^{3}$ De Man propone que los siguientes elementos hegelianos son predominantes los estudios literarios del siglo XX: Historia literaria como articulación entre la era Helénica y la Cristiana, o entre el mundo Hebráico y el Helénico; La sistematización entre distintas formas y géneros artísticos como modos determinados de representación; y la concepción de la periodización histórica como desarrollo, progresivo o regresivo, de una conciencia colectiva.

4 "La belleza estética como una manifestación externa de un contenido ideal que es, en sí mismo, una experiencia interiorizada, la emoción recolectada de una percepción pasada" (DE MAN, 1996, 100).
} 
Man extrema esta argumentación al afirmar que Hegel es el teórico de la internalización (en el recorrido que va desde la Fenomenología del espíritu a la Estética).

Nos detenemos en esta argumentación porque De Man involucra allí las dos versiones de la "memoria" que vimos en el apartado "Representación" de la Enciclopedia: nos referimos a la distinción entre "Erinnerung" y "Gedachtnis" - la primera se traduce por "recolección" o "recuerdo", mientras que la segunda se traduce por "memoria"-. De Man destaca la importancia de la "Erinnerung como base de la estética así como también de la conciencia histórica. Erinnerung, recolección, como acopio interno y la preservación de la experiencia, reúne historia y belleza en la coherencia de un sistema" (101). Ahora bien, si el arte -definido como la manifestación sensible de la Idea- ocurre en el modo de la recolección, es decir, como un proceso dialéctico que articula interioridad y exterioridad, De Man se pregunta "¿en qué parte, dentro del sistema hegeliano, puede decirse que el intelecto, la mente, o la idea dejan rastros/huellas [traces] sobre el mundo, y cómo tiene lugar esta aparición sensorial?" (101). Como veremos a continuación, este lugar lo va a ocupar el significante, la letra, como inscripción material.

Entonces, si "Erinnerung" se relaciona con la memoria entendida como recolección de recuerdos, "Gedachtnis" por su parte es la facultad de la mente que apunta al acto de "memorización" . Dice Hegel: "en el discurso ordinario [opuesto al filosófico] a menudo se la confunde con Erinnerung [recolección], y con representación e imaginación”. (2005, 500) De Man destaca la importancia de la memorización en relación con el pensamiento -que es precisamente el lugar de la contradicción que retomamos de Surber-:

La sorpresa, en Hegel, es que la progresión desde la percepción al pensamiento depende de la facultad mental de memorización [...]. Para entender el pensamiento, primero tenemos que entender la memoria pero, dice Hegel, "entender el lugar y el significado de la memoria en el estudio sistemático del intelecto [...] es uno de los puntos más difíciles en el estudio de la mente'(De Man, 1999, 101).

La memorización entonces se relaciona con los signos: la escritura aparece a posteriori como signo de signos. En el parágrafo 460 de la Enciclopedia Hegel establece que el nombre es "el enlace de la representación con algo interior, con la intuición como algo exterior, es el mismo exterior. El recuerdo de la exterioridad es la memoria" (HEGEL, 2005, 505). De esta manera, es en el nombre donde ocurre el pensamiento; los nombres no como objetos del pensamiento sino como el medio donde pensamos la cosa, el objeto. Dice Hegel: "es en el nombre donde pensamos" (HEGEL, 2005, 506) ${ }^{5}$. La escritura como máquina del pensamiento.

Memorización (Gedachtnis) se distingue así de recolección (Erinnerung) e imaginación (Phantasie) porque es sin imagen (bildlos), pero igualmente posee materialidad. Para Hegel, aprender es "Aus-wendig lernen", "to learn something by heart", "aprender de memoria", es decir, aprender por el registro de la materialidad, cuando el significado se borra y ya no se lo asocia con una palabra. Recuperamos a continuación un pasaje extenso de Hegel sobre este tema, ya que se conecta con lo que vimos en Surber y también funciona como referencia para la tesis principal de Paul de Man en el texto que veníamos analizando:

alguien sabe un texto de memoria solamente cuando las palabras no poseen ningún sentido [...] La memoria es de esta manera el paso a la actividad del pensamiento que ya no tiene ninguna significación, esto es, lo subjetivo ya no es algo distinto de su objetividad,

\footnotetext{
${ }^{5}$ El traductor de la edición de la Enciclopedia con la que trabajamos, recupera en nota al pie la frase original en alemán "Es ist in Namen, daß wir denken" que literalmente significa: "es en (los) nombres que pensamos" (HEGEL, 2005, 506)
} 
igual que esta interioridad está-siendo en ella misma [...] La memoria como tal es de suyo la manera meramente exterior, el momento unilateral de la existencia del pensamiento (HEGEL, 2005, 509).

A partir de esto, Paul de Man responde la pregunta que se había planteado sobre la aparición sensorial de la Idea: ésta ocurre entonces en la memorización de un nombre en tanto que es inseparable de su notación, inscripción material, escritura. "La idea, en otras palabras, hace su aparición sensorial, en Hegel, como la inscripción material del nombre. El pensamiento depende enteramente de una facultad mental que es completamente mecánica, tan carente como sea posible de los sonidos y las imágenes de la imaginación, o de la mina oscura de la recolección [Erinnerung], que yace más allá del alcance de las palabras y del pensamiento" (DE MAN, 1996, 102). La memoria como Gedachtnis es el punto donde se sitúa la apuesta demaniana de que lo "bello" no es la manifestación sensible de la Idea, sino su inscripción material. Para De Man, hay memoria en la medida en que se olvida el recuerdo de la intuición y se alcanza la exterioridad mecánica de la palabra. Pensamos en los nombres pero ellos son, en tanto signos, "la inscripción muda [silent] donde la relación entre lo que uno percibe y lo que comprende, entre letra escrita y significado, es sólo exterior y superficial" (102), lo cual difiere completamente de la noción de símbolo como garante del goce estético que referimos anteriormente. Si volvemos a las dos afirmaciones de Hegel sobre el arte, la explicación de De Man es que el hecho de que el arte sea cosa del pasado, y que sea simbólico, equivalen a la misma afirmación:

En la medida en que el paradigma del arte es el pensamiento más que la percepción, el signo más que el símbolo, escribir más que pintar o la música, también va a ser memorización más que recolección. Como tal, pertenece de hecho al pasado que, en palabras de Proust, nunca puede ser capturado, retrouvé. El arte es cosa del pasado en un sentido radical, porque, como la memorización, deja la interiorización de la experiencia siempre detrás suyo. El arte pertenece al pasado en tanto inscribe materialmente, y así olvida, su contenido ideal (DE MAN, 1996, 103).

Como se puede ver, mediante el desarrollo de la tensión entre "memorización" y "recolección" De Man toma partido por el signo en detrimento del símbolo para problematizar el lugar que ocupa la estética en el pensamiento hegeliano. Si lo bello, en tanto manifestación sensible de la Idea, toma la forma del "símbolo", ¿existe en Hegel un tipo de arte no simbólica y, por tanto, no estética, que tome la forma de lo que describimos como "signo"? Recuperamos la respuesta de De Man a este interrogante, no sólo porque concluye la argumentación de su texto, sino también porque consideramos que es una buena herramienta para pensar varias de las relecturas que se hicieron de Hegel a partir del siglo XX - entre las que se encuentran las intervenciones que analizamos en este trabajo-.

La apuesta demaniana consiste en afirmar que en Hegel la teoría del signo se manifiesta en las formas de arte "feas", es decir, no estéticas, entre las cuales la "alegoría" es la principal. La alegoría, al igual que la parábola o la imagen, son considerados géneros inferiores porque no presentan, como sí lo hace el símbolo, los significados siguiendo su adecuación a la realidad. Podemos decir que la inversión dialéctica es la lógica que rige todo el texto de De Man, revalorizando la mecanicidad del signo y la memorización por sobre el aura estética del símbolo y la recolección. Sobre el final del texto leemos: "los costados negados del canon hegeliano son quizás articulaciones maestras en lugar de los muy llamativos juicios sintéticos que recordamos como los lugares comunes de la historia del siglo XIX" (DE MAN, 1996, 104). Esto se conecta con la hipótesis central de Paul de Man, quien afirma que la filosofía de Hegel "es de hecho una alegoría 
de la disyunción entre filosofía e historia, entre literatura y estética, entre experiencia literaria y teoría literaria" (104). Teniendo en cuenta lo visto hasta ahora, proponemos interpretarlo de la siguiente manera: la dialéctica, mientras cuenta la historia de la síntesis y la superación, realiza performativamente su disyunción, es decir, la interrupción de su poder de síntesis. Acordamos con Paul de Man en cuanto a que el motivo de esta disyunción no es historizable, en la medida en que son razones "inherentes al lenguaje, en su necesidad que también es imposibilidad, de conectar al sujeto con su predicado o al signo con sus significaciones simbólicas" (DE MAN, 1996, 104). El hecho de que esta disyunción sea inherente al lenguaje se conecta con una de las sentencias más recordadas de De Man sobre la resistencia a la teoría, pues justamente establece que es una "resistencia inherente a la empresa teórica misma" (DE MAN, 2002, 12) $)^{6}$. De esta manera, proponemos que la resistencia es el modo en el que se manifiesta la tensión entre experiencia y teoría, es decir, entre experiencia y lenguaje.

\footnotetext{
6 "La resistencia a la teoría es una resistencia al uso del lenguaje sobre el lenguaje [...] una resistencia al lenguaje mismo o a la posibilidad de que el lenguaje contenga factores o funciones que no puedan ser reducidos a la intuición" (2002, 12-13). La categoría de "resistencia a la teoría" fue concebida por Paul de Man a mediados de los años ochenta, como resultado de su disputa con el New Criticism estadounidense. De Man polemiza con las lecturas críticas que intentan explicar la literatura a partir de referencias exclusivas a su dimensión histórica o a su dimensión estética
}

CORTÉS, Federico Gabriel. El problema del lenguaje en Hegel: tres lecturas de la Enciclopedia de las ciencias filosóficas (1817). Griot : Revista de Filosofia, Amargosa - BA, v.21 n.3, p.258-267, outubro, 2021. 


\section{Referencias}

DE MAN, Paul. Aesthetic ideology. Minneapolis: Yale University Press, 1996.

DE MAN, Paul. The Resistance to Theory. Minneapolis: University of Minnnesota Press, 2002.

HEGEL, George Wilhelm. Enciclopedia de las ciencias filosóficas en compendio. Madrid: Alianza Editorial., 2005.

HEGEL, George Wilhelm (2011). Ciencia de la lógica. Madrid: Abada Editores, 2011.

PRÓSPERI, Germán Osvaldo. La respiración del Ser. Apnea y ensueño en la filosofía hegeliana. Buenos Aires: Miño y Davila Editores, 2018.

STERN, David (Ed.). Essays on Hegel's Philosophy of Subjective Spirit. Albany: State University of New York Press, 2013.

Autor(a) para correspondência / Corresponding author: Federico Gabriel Cortés. fede.gcortes@gmail.com 